\title{
Noise-Assisted Microwave Up-conversion by Vortices in Thin-Film Superconductors with a dc-Biased Washboard Pinning Potential
}

\author{
Valerij A. Shklovskij • Oleksandr V. Dobrovolskiy • \\ Michael Huth
}

Received: 31 October 2012 / Accepted: 1 December 2012 / Published online: 20 December 2012

(C) Springer Science+Business Media New York 2012

\begin{abstract}
So far the main theoretical basis for understanding and optimization of the microwave properties of vortices in type II superconductors has been relying upon the Coffey-Clem (CC) approach for the linear impedance at nonzero temperature. However, the $\mathrm{CC}$ model does not account for the non-linear response and the possibility to control it by changing the value of the dc transport current in a superconducting sample. For this reason, we have exactly solved the Langevin equation for the two-dimensional nonlinear vortex dynamics in a dc bias-tilted cosine pinning potential in the presence of an ac current of arbitrary amplitude and frequency $\omega$ and have, thereby, substantially generalized the $\mathrm{CC}$ results. In this work we analyze the behavior of the non-linear response on $k \omega$-frequency in a wide range of dc and ac current densities, $\omega$, and temperature. The $k \omega$ response is found to depend strongly on all these parameters, as exemplified for the third-harmonic $(k=3)$ transformation coefficient $Z_{3}$. The parametric window for the most enhanced up-conversion is presented. The predicted effects can be experimentally verified in thin-film superconductors with some pinning potential of the washboard type.
\end{abstract}

\footnotetext{
V.A. Shklovskij

Institute for Theoretical Physics, NSC-KIPT, 61108 Kharkov, Ukraine

\section{V.A. Shklovskij}

Department of Physics, Kharkiv National University, 61077

Kharkov, Ukraine

O.V. Dobrovolskiy $(\bowtie) \cdot$ M. Huth

Physikalisches Institut, Goethe-Universität, 60438 Frankfurt am Main, Germany

e-mail: Dobrovolskiy@Physik.uni-frankfurt.de
}

Keywords Microwave up-conversion · Non-linear impedance $\cdot$ Washboard pinning potential $\cdot$ Generation of higher harmonics

\section{Introduction}

The understanding and optimization of the non-linear microwave properties of vortices are important both for basic physics and applications [1]. One of the most valuable experimental methods for the investigation of flux pinning mechanisms and vortex dynamics in type-II superconductors is the measurement of the complex ac response in the radiofrequency and microwave range [2-5]. Very early experimental data on the power absorption by vortices in PbIn and NbTa films over a wide frequency range have been obtained by Gittleman and Rosenblum (GR) [2] and interpreted on the basis of their model which considers small oscillations of damped vortices in a harmonic pinning potential (PP) at zero temperature. The success of the GR model stimulated attempts to apply it for high temperature superconductors (HTSCs) where the effects of thermal agitation are especially important due to relatively low pinning activation energies and the high critical temperatures.

As the GR model was developed for zero temperature and could not account for the thermally activated flux flow (TAFF) and creep, which both are very pronounced in HTCSs [6], a need arose for a more general model of the ac vortex dissipation at different temperatures and frequencies. To accomplish this, GR's equation of motion for a vortex was in the following supplemented by Coffey and Clem (CC) [7] with a thermofluctuation force being a Gaussian white noise with zero mean and a cosine PP for taking into account the possibility of vortex hopping between different potential wells. In the limit of small ac current in the 
absence of any dc bias, i.e., for a nontilted cosine PP, CC solved this new equation of motion by a scalar continuedfraction expansion [7] using the analogy between a pinned vortex and the motion of a Brownian particle in a periodic potential.

A comparative overview on the complete data treatment in a typical microwave experiment can be found in Ref. [3]. In that work [3] the theoretical treatment on the basis of the GR, CC, and some other models is summarized. Experimentally, a very sensitive way to study superconducting nonlinearities is monitoring the higher harmonics electric field response to an ac current of given frequency. For instance, harmonic measurements were performed on superconducting films and crystals to study the microwave non-linear behavior as a function of temperature and external magnetic field [8-12]. This is why the examination of a strong $(\mathrm{ac}+\mathrm{dc})$-driving is especially interesting both for the theory and a number of high-frequency and microwave applications. This evidently requires one to consider a PP strongly tilted by a dc bias and subjected to an ac current of arbitrary amplitude and frequency.

For this reason, the two-dimensional Langevin equation for the non-linear temperature-dependent guided vortex motion in a tilted cosine washboard pinning potential (WPP), supplemented with the Hall term, was considered [13] at arbitrary dc $j_{0}$ and ac $j_{1}$ densities, frequency $\omega$, and current flow at an arbitrary angle $\alpha$ with regard to the WPP's guiding direction. This problem has been exactly solved [13] in terms of matrix continued fractions, providing expressions for experimentally detectable $\mathrm{dc}$ and ac responses. In particular, it has been shown how pronounced non-linear effects [14], such as Shapiro-like steps [15] and phase-locking features [16], appear in response to $(\mathrm{dc}+\mathrm{ac})$-driving and why the response on $k \omega$-frequencies, as generic to the non-linear systems, is to be expected [13].

As will be shown in this work, within a certain range of $j_{0}, j_{1}, \omega$, and temperature, two further unique features of the system under consideration are (i) an enhancement of the real part of the $k$ th transformation coefficients $\operatorname{Re} Z_{k}$ which corresponds to the up-conversion and (ii) the evidence of $\operatorname{Re} Z_{k}<0$ having the physical meaning of the generation of a higher harmonic electric field contribution. Both these features are proposed to be investigated experimentally on thinfilm superconductors with some PP of the washboard type when subjected to superimposed ac and dc current drives, e.g., in the spirit of Ref. [5]. Both these issues are addressed next.

\section{Formulation and Solution of the Problem}

Neglecting the Hall effect, the Langevin equation for a vortex moving with velocity $\mathbf{v}$ in a magnetic field $\mathbf{B}=\mathbf{n} B$
$(B \equiv|\mathbf{B}|, \mathbf{n}=n \mathbf{z}, \mathbf{z}$ is the unit vector in the $z$ direction and $n= \pm 1)$ has the form $[13,16]$

$\eta \mathbf{v}=\mathbf{F}_{\mathrm{L}}+\mathbf{F}_{\mathrm{p}}+\mathbf{F}_{\text {th }}$,

where $\mathbf{F}_{\mathrm{L}}=n\left(\Phi_{0} / c\right) \mathbf{j} \times \mathbf{z}$ is the Lorentz force with $\Phi_{0}$ being the magnetic flux quantum, while $c$ is the speed of light. $\mathbf{j}=\mathbf{j}_{0}+\mathbf{j}_{1} \exp i \omega t$, where $\mathbf{j}_{0}$ and $\mathbf{j}_{1}$ are the dc and ac current density amplitudes and $\omega$ is the angular frequency. In Eq. (1) $\mathbf{F}_{\mathrm{p}}=-\nabla U_{\mathrm{p}}(x)$ is the anisotropic pinning force, $U_{\mathrm{p}}(x)=\left(U_{\mathrm{p}} / 2\right)(1-\cos k x)$ with $k=2 \pi / a$ is the periodic WPP, where $U_{\mathrm{p}}$ and $a$ are its depth and period, respectively. $\mathbf{F}_{\text {th }}$ is the thermal fluctuation force represented by a Gaussian white noise with zero mean and $\eta$ is the vortex viscosity. To simplify the subsequent analysis we consider the current flow along the WPP "channels" $\left(\alpha=0^{\circ}\right)$ and, thus, the vortices' movement perpendicular to their direction. In this case, the stationary ac electric field $E \equiv E\left(j_{0}, j_{1}, \omega, g\right)$, measured along the WPP channels and being independent of the initial conditions, is the sum of $\omega$-harmonics [13]

$E=n \rho_{f} j_{\mathrm{c}} \sum_{k=1}^{\infty} j_{1}^{k} \operatorname{Re}\left\{Z_{k}(\omega) e^{i k \omega t}\right\}$

where

$Z_{k}(\omega)=\delta_{1, k}-i \psi_{k}(\omega) / j_{1}^{k}$

The dimensionless transformation coefficients $Z_{k}$ have the physical meaning of the $k$ th harmonics of the input frequency $\omega$ in the non-linear ac response $E\left(j_{0}, j_{1}, \omega, g\right)$. The functions $\psi_{k}(\omega)$ are determined in terms of matrix continued fractions [13], $\delta_{1, k}$ is Kronecker's symbol, and $\rho_{\mathrm{f}} \equiv$ $B \Phi_{0} / \eta c^{2}$ is the flux-flow resistivity.

Below, both the dc and ac amplitudes are calculated in units of $j_{\mathrm{c}} \equiv c U_{\mathrm{p}} k / 2 \Phi_{0}$. Here $j_{\mathrm{c}}$ is the crossover current density corresponding to the middle point of a sharp step-like non-linear transition from the TAFF regime in the vortex dynamics to that of the viscous FF [13]. The frequency $\omega$ is expressed in units of the depinning frequency $\omega_{\mathrm{p}} \equiv U_{\mathrm{p}} k^{2} / 2 \eta[13]$. In the absence of a dc drive, $\omega_{\mathrm{p}}$ separates the low-frequency regime, where the pinning forces dominate and the non-linear impedance $Z(\omega)$ is mainly nondissipative, from the high-frequency regime where the frictional forces dominate and $Z(\omega)$ is dissipative [2]. Quantitatively, an experimentally estimated value of $\omega_{\mathrm{p}}$ in the absence of a dc current at a temperature of $\simeq 0.6 T_{\mathrm{c}}$ is $\omega_{\mathrm{p}} \approx$ $7 \mathrm{GHz}$ for a $20 \mathrm{~nm}$-thick [17] or a $40 \mathrm{~nm}$-thick [18] $\mathrm{Nb}$ film. For comparison, in YBCO $\omega_{\mathrm{p}}<15 \mathrm{GHz}$, typically [1]. While plotting the data, the dimensionless inverse temperature $g=U_{\mathrm{p}} / 2 T$ will be used for convenience as it describes the relative intensity of thermal fluctuations with respect to the PP depth. A typical experimental value of $U_{\mathrm{p}}$ in $\mathrm{Nb}$ films is $\simeq 5000 \mathrm{~K}$ [19], while it is $\simeq 1000 \mathrm{~K}$ [20] in YBCO. 


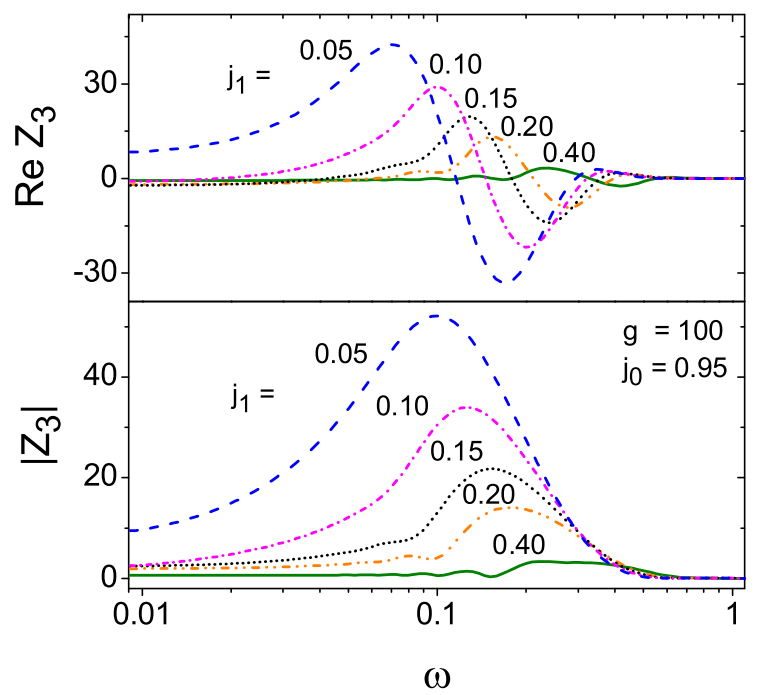

Fig. $1 \operatorname{Re} Z_{3}$ and $\left|Z_{3}\right|$ versus frequency $\omega$ for dc bias $j_{0}=0.95$, inverse temperature $g=100$, and a series of ac amplitudes $j_{1}$, as indicated

\section{Discussion}

As usually investigated in experiments [8-12], we discuss the third-harmonic voltage response considering the frequency, dc bias, ac amplitude and temperature dependences of the real part of the transformation coefficient $\operatorname{Re} Z_{3}$ and its absolute value $\left|Z_{k}\right|=\sqrt{\left(\operatorname{Re} Z_{3}\right)^{2}+\left(\operatorname{Im} Z_{3}\right)^{2}}$. It is worth noticing that similar peculiarities are generic to all $k$ th transformation coefficients.

In Fig. 1 we plot the frequency dependences $\operatorname{Re} Z_{3}(\omega)$ and $\left|Z_{3}(\omega)\right|$ for $j_{0}=0.95$ as representative for close-tocritical dc biases $j_{0}<1$, subcritical ac amplitudes $j_{1}<1$, and moderately low temperatures $g=100$. As evident from the figure, $\operatorname{Re} Z_{3}(\omega)$ has a wave form, whereas the curves $\left|Z_{3}(\omega)\right|$ resemble that of a bell. Both the responses tend to zero as $\omega \rightarrow 0$ or $\omega \rightarrow 1$ and are substantially nonmonotonic at intermediate frequencies $\omega \simeq 0.1$. Furthermore, multiple sign reversals are detectable in the curves $\operatorname{Re} Z_{3}(\omega)$ for $\omega=0.1 \div 0.3$. For this reason, all the data below are plotted for $\omega=0.2$.

The negative sign in the real part of the non-linear impedance $\operatorname{Re} Z_{1}$ on the ac frequency $\omega$ has been previously reported [21]. Similarly to the problem of resistively shunted non-linear Josephson junction [22], in our case finite temperature overcritical biases can also lead to a sign change in the real part of the impedance. In the previous communication [21] it has been shown that such an anomalous impedance behavior is more pronounced for low temperatures and small ac amplitudes and is challenging with regard to the development of parametric amplifiers and improving efficiency of down converters operating with Abrikosov vortices. In the higher harmonics responses $\operatorname{Re} Z_{k}(\omega)$, this

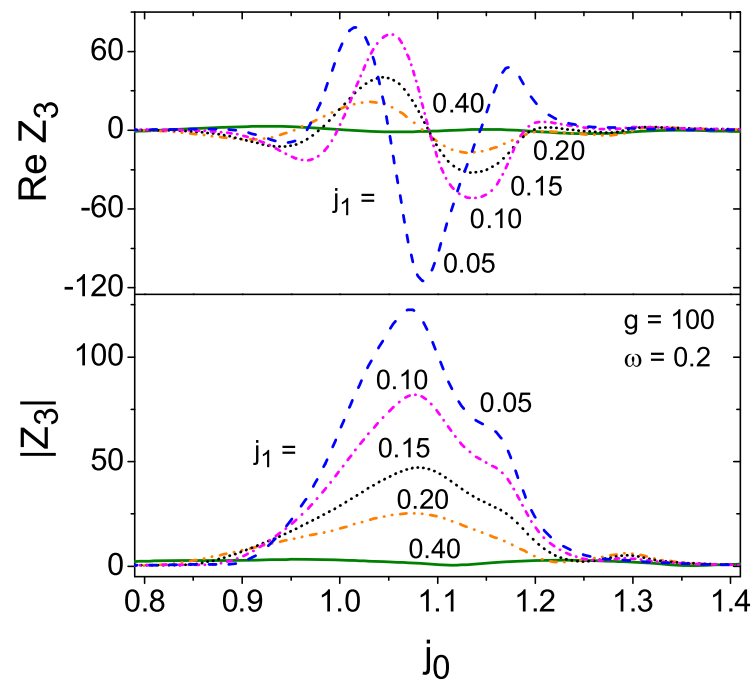

Fig. $2 \operatorname{Re} Z_{3}$ and $\left|Z_{3}\right|$ versus dc bias $j_{0}$ for the frequency $\omega=0.2$, the inverse temperature $g=100$, and a series of ac amplitudes $j_{1}$, as indicated. The peculiarities are more prominent at close-to-critical dc biases $j_{0} \simeq 1$

may cause a peak in the vicinity of $j_{0}=1$ as depicted in Fig. 2. That is, both the dependences $\operatorname{Re} Z_{3}(\omega)$ and $\left|Z_{3}(\omega)\right|$ are non-monotonic, with a series of extrema. The magnitude of any extremum is increasing with decreasing $j_{1}$ and increasing $g$.

From Fig. 2 it follows that larger absolute values of $\operatorname{Re} Z_{3}$ and $\left|Z_{3}\right|$ ensue at close-to-critical dc biases $0.9 \lesssim j_{0} \lesssim 1.3$. From the mathematical viewpoint, this circumstance can be connected with the strong enhancement of the effective diffusion coefficient $D$ of an overdamped Brownian particle in a tilted WPP near the critical tilt [23]. This enhancement of $D$, in turn, relates to the resonance-activated reduction in the mean escape time of the Brownian particle due to an oscillatory variation of the pinning barrier height [24] which can also be inherited by higher harmonics responses.

A most curious behavior is demonstrated by the curves $\operatorname{Re} Z_{3}$ and $\left|Z_{3}\right|$ as functions of the inverse temperature $g$, see Fig. 3. A number of peaks in $\operatorname{Re} Z_{3}(g)$ and $\left|Z_{3}\right|(g)$ is a manifestation of the stochastic resonance [25], usually defined as the phenomenon of amplification of a small ac signal in a periodic potential with the help of noise [26]. Here, the appearance of an enhanced $3 \omega$ signal represents a noise-assisted microwave up-conversion which can be efficiently controlled by the appropriate choice of the parameters $j_{0}, j_{1}, \omega$, and $g$.

For completeness, we consider $\operatorname{Re} Z_{3}$ and $\left|Z_{3}\right|$ as functions of the ac amplitude $j_{1}$. As evident from Fig. 4 , the above-mentioned anomalies in $\operatorname{Re} Z_{3}$ and $\left|Z_{3}\right|$ are expected when $j_{1} \lesssim 0.5$, i.e., must be investigated under a small microwave excitation $j_{1} \ll 1$. 


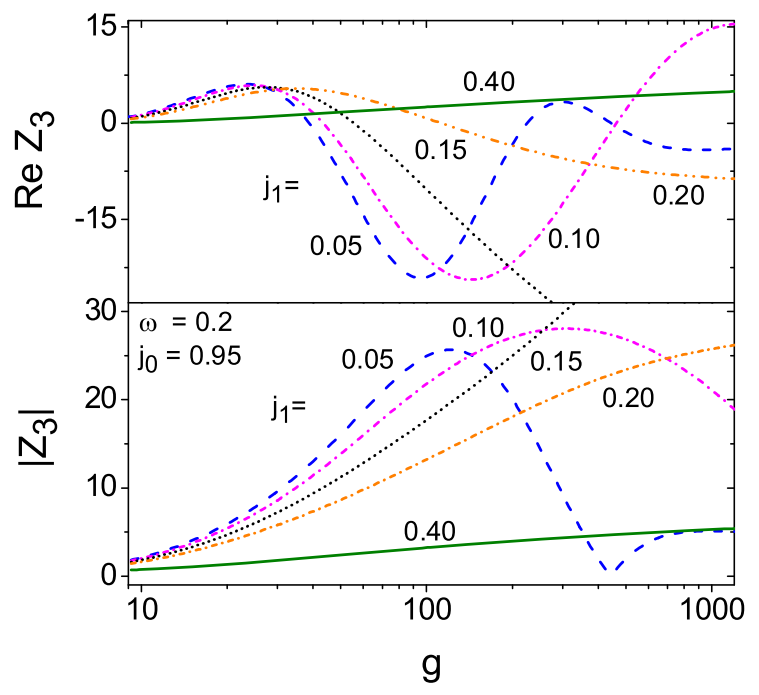

Fig. $3 \operatorname{Re} Z_{3}$ and $\left|Z_{3}\right|$ versus inverse temperature $g$ for the frequency $\omega=0.2$, the dc bias $j_{0}=0.95$, and a series of ac amplitudes $j_{1}$, as indicated. An extremum in either curve is caused by the phenomenon of stochastic resonance and substantiates the enhanced microwave upconversion by vortices at a certain temperature value

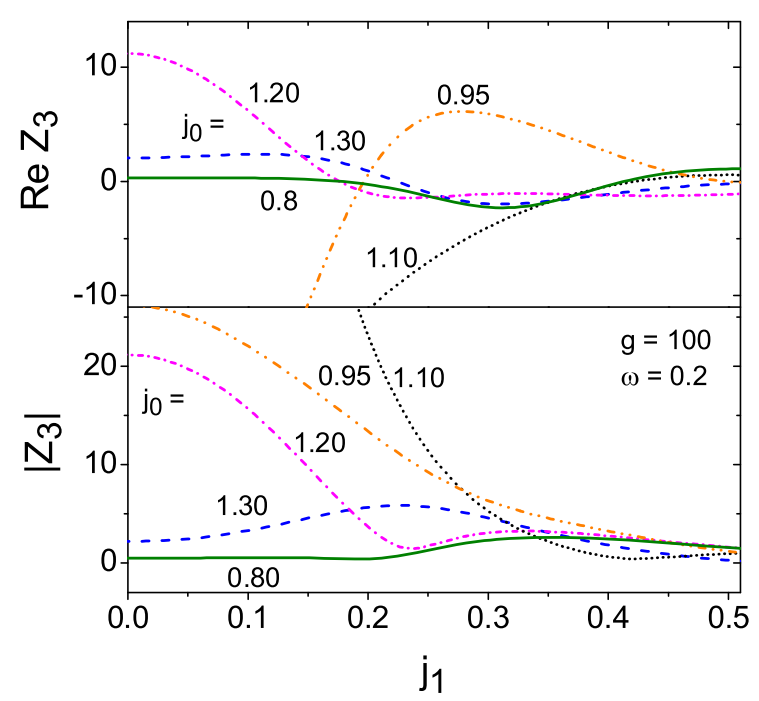

Fig. $4 \operatorname{Re} Z_{3}$ and $\left|Z_{3}\right|$ versus microwave amplitude $j_{1}$ for the frequency $\omega=0.2$, the inverse temperature $g=100$, and a series of $\mathrm{dc}$ biases $j_{0}$, as indicated

\section{Conclusion}

We have theoretically investigated the $3 \omega$ electric field response of a thin-film superconductor with a WPP in a wide range of dc biases $j_{0}$, microwave amplitudes $j_{1}$, ac frequencies $\omega$, and (inverse) temperature $g$. Two main results have been obtained. (i) A noise-enhanced up-conversion is predicted in the microwave range $\omega \simeq 0.2 \omega_{\mathrm{p}}$ for $j_{0}<j_{\mathrm{c}}$, $j_{1} \ll j_{\mathrm{c}}$, and relatively low temperatures $T \simeq 0.005 U_{\mathrm{p}}$ with respect to the pinning activation energy. (ii) The genera- tion of higher $\omega$-harmonics is predicted at certain values of $j_{0}, j_{1}, \omega$, and $g$.

Concerning the general validity of the results obtained, it should be noted that Eq. (2) is valid only at small magnetic fields $B \ll B_{\mathrm{c} 2}$, as the Langevin equation (1) has been exactly solved only in the single-vortex approximation, such that no vortex-vortex interactions are captured in the model considered hear. In this work, we have limited our analysis to the case when both dc and ac currents are applied parallel to the WPP channels $(\alpha=0)$ just to simplify the presentation of the data, though any arbitrary angles $\alpha \neq 0$ can be accounted for on the basis of our approach [13]. This is particularly valuable for fitting the experimental data measured on nanostructured samples, where during the lithographic process a small misalignment of the microbridge structures with respect to the WPP channels cannot be excluded, i.e., the angle $\alpha$ may differ by a small amount $\delta \alpha$ from its nominal value [19].

The experimental realization of the model in thin-film geometry opens up the possibility for a study of higher harmonics responses under (dc+ac)-driving by measuring, for instance, ac power absorption. Experimental control of the frequency and magnitude of the driving forces, damping, pinning parameters, and temperature can be effectively provided. One of the most crucial issues for the experiment is to adequately superimpose the applied currents and thereafter, to uncouple the picked-up dc and microwave signals maintaining the matching of the impedances of the line and the sample.

Recently, the frequency dependence of the dc voltage at large ac amplitudes in the frequency range between $0.5 \mathrm{MHz}$ and $2 \mathrm{GHz}$ has been experimentally studied [4] on $\mathrm{Pb}$ films with a nanoengineered array of asymmetric pinning sites and the temperature dependence of the output power with and without dc bias has been measured [5] by using combined $\mathrm{dc}$ and microwave ac electronic measurements on (sub-)micron-patterned high- $T_{\mathrm{c}}$ films for $\omega=0.4 \div 8 \mathrm{GHz}$. We consider these systems $[4,5]$ to be most promising for experimental verification of the results presented here.

Acknowledgements O.V.D. gratefully acknowledges financial support by the Deutsche Forschungsgemeinschaft (DFG) through Grant No. DO 1511/2-1.

\section{References}

1. Silva, E., Pompeo, N., Sarti, S., Amabile, C.: Vortex State Microwave Response in Superconducting Cuprates, pp. 201-243. Nova Science, Hauppauge (2006), Chap. 1

2. Gittleman, J.I., Rosenblum, B.: Phys. Rev. Lett. 16, 734 (1966)

3. Pompeo, N., Silva, E.: Phys. Rev. B 78, 094503 (2008)

4. Jin, B.B., Zhu, B.Y., Wördenweber, R., de Souza Silva, C.C., Wu, P.H., Moshchalkov, V.V.: Phys. Rev. B 81, 174505 (2010)

5. Wördenweber, R., Hollmann, E., Schubert, J., Kutzner, R., Panaitov, G.: Phys. Rev. B 85, 064503 (2012) 
6. Blatter, G., Feigel'man, M.V., Geshkenbein, V.B., Larkin, A.I., Vinokur, V.M.: Rev. Mod. Phys. 66, 1125 (1994)

7. Coffey, M.W., Clem, J.R.: Phys. Rev. Lett. 67, 386 (1991)

8. Wilker, C., Zhi-Yuan, S., Pang, P., Holstein, W.L., Face, D.W.: IEEE Trans. Appl. Supercond. 5, 1665 (1995)

9. Willemsen, B.A., Kihlstrom, K.E., Dahm, T., Scalapino, D.J., Gowe, B., Bonn, D.A., Hardy, W.N.: Phys. Rev. B 58, 6650 (1998)

10. Gallitto, A., Vigni, M.L.: Physica C, Supercond. 305(1-2), 75 (1998)

11. Booth, J., Vale, L., Ono, R.: IEEE Trans. Appl. Supercond. 11, 1387 (2001)

12. Pestov, E., Nozdrin, Y., Kurin, V.: IEEE Trans. Appl. Supercond. 11, 131 (2001)

13. Shklovskij, V.A., Dobrovolskiy, O.V.: Phys. Rev. B 78, 104526 (2008)

14. Shklovskij, V.A., Dobrovolskiy, O.V.: J. Phys. Conf. Ser. 150(5), 052241 (2009)

15. Shklovskij, V.A.: J. Phys. Conf. Ser. 150(5), 052240 (2009)

16. Shklovskij, V.A., Dobrovolskiy, O.V.: Phys. Rev. B 84, 054515 (2011)
17. Pompeo, N., Silva, E., Sarti, S., Attanasio, C., Cirillo, C.: Physica C, Supercond. 470(19), 901 (2010)

18. Janjušević, D., Grbić, M.S., Požek, M., Dulčić, A., Paar, D., Nebendahl, B., Wagner, T.: Phys. Rev. B 74, 104501 (2006)

19. Soroka, O.K., Shklovskij, V.A., Huth, M.: Phys. Rev. B 76, 014504 (2007)

20. Palstra, T.T.M., Batlogg, B., Schneemeyer, L.F., Waszczak, J.V.: Phys. Rev. Lett. 61, 1662 (1988)

21. Shklovskij, V.A., Dobrovolskiy, O.V., Huth, M.: In: Conference Program SCC-2011, p. 85 (2011)

22. Coffey, W.T., Déjardin, J.L., Kalmykov, Y.P.: Phys. Rev. B 62, 3480 (2000)

23. Reimann, P., Van den Broeck, C., Linke, H., Hänggi, P., Rubi, J.M., Pérez-Madrid, A.: Phys. Rev. Lett. 87, 010602 (2001)

24. Boguñá, M., Porrà, J.M., Masoliver, J., Lindenberg, K.: Phys. Rev. E 57, 3990 (1998)

25. Gammaitoni, L., Hänggi, P., Jung, P., Marchesoni, F.: Rev. Mod. Phys. 70, 223 (1998)

26. Jung, P., Hänggi, P.: Phys. Rev. A 44, 8032 (1991) 